\title{
On the Influence of Tools on Collaboration in Participative Enterprise Modeling - An Experimental Comparison between Whiteboard and Multi-Touch Table
}

\author{
Anne Gutschmidt \\ University of Rostock, Department of Computer Science \\ Rostock, Germany
}

anne.gutschmidt@uni-rostock.de

\begin{abstract}
The paper presents an experiment about the influence of the modeling tool on group work in the context of enterprise modeling. A goal modeling task was set where three groups of three persons worked with a whiteboard, and three groups of three persons worked with a multi-touch table. Comparisons of working styles between the two tools indicate that multi-touch tables promote parallel working and that a team member's position plays a role in taking on certain tasks. Whiteboard users may more easily lose track of what teammates are doing.

Keywords: enterprise modeling, participative, multi-touch table, group work, experiment
\end{abstract}

\section{Introduction}

Enterprise modeling is a powerful way of capturing important information about a company, such as structures, processes and dependencies. It enables a company to identify problems and their causes as well as potential for change, and helps to prepare for and implement such changes [1]. When creating an enterprise model, usually comprising several intertwined submodels, it is necessary to involve all stakeholders. Participative enterprise modeling suggests that the modeling is performed by the stakeholders themselves with the support of facilitators representing the experts of the modeling method [2]. The stakeholders are domain experts that directly provide the necessary knowledge in the modeling process.

Multi-touch tables represent useful tools to support enterprise modeling performed in a group. In contrast to modeling at a whiteboard or with pen and paper, content can be easily changed and deleted, and, what is of greatest advantage, models can be saved digitally, shared and reused at any time. The differences in handling this tool may also cause differences in the way a group works together. In this paper, a study is presented which examines the influence of tool on the way groups work together. We compared a multi-touch table (MTT) with a whiteboard, the latter representing a traditional tool. We focused on the following research questions: (1) How and to what extent do single team members contribute to the modeling task? This should also show how evenly distributed individual contributions are, depending on the tool. (2) Are there different working styles depending on the tool? The latter question concerns aspects such as task division and coordination. (3) Are there any differences in team performance depending on the tool? The goal of this research is to find out whether the MTT already provides advantages that must be taken into consideration when deciding on a modeling tool. Furthermore, we wanted to look for hints on working styles of whiteboard users that might be transferred to the MTT, e.g. by aspects of function and design of modeling software for the MTT.

In the next section, some background on group work will be presented showing relations to participative enterprise modeling. In the third section, the method of the study is presented, followed by results in section 4 . The last section concludes the paper with a discussion of the results, including limitations of the study and implications for future research. 


\section{State of the Art}

\subsection{Group Work and Participative Enterprise Modeling}

The main reason why we work in groups is that we hope to perform better by gathering performance and ability of several individuals. However, group work may bring both increase and decrease in performance with regard to motivation, individual skills and coordination (see e.g. [3] for more information). E.g. the mere presence of other persons can motivate someone to put more effort in a task. Furthermore, in some teams, persons compensate weaker performances of their teammates $[3,4]$. On the other hand, persons might be less motivated because they do not see the concrete value of their contribution in the team work [5]. With regard to individual skill, performance decrease might occur because teammates interrupt the flow of ideas of a person by keeping on voicing their own ideas. However, being inspired by others' ideas may also lead to new ideas and thus a performance increase [3]. Group work, of course, also requires more effort on coordination the larger a group is, e.g., there has to be an agreement on who is allowed to talk at a certain moment. [6] showed that people produced less ideas in a brainstorming task when working in a group than when working individually.

Participative enterprise modeling represents classic group work which is just as prone to the above-mentioned effects. It involves a variety of activities which the members of the group must perform. Firstly, it involves gathering knowledge and ideas which requires a significant effort on coordinating the contributions of all group members. Secondly, situations may occur when one of several alternatives has to be chosen by the group. If a person is dominating this decision process because of their knowledge or intelligence, this might lead to good overall performance. However, if such dominance is based on characteristics such as the rank of a group member, this might deteriorate results and lead to a decrease in the motivation of other team members to voice their ideas. Especially when tasks are at hand where there is no complete knowledge and the group has to base decisions on guesses, more extroverted persons may possibly dominate discussions at the expense of the result's quality (see [1] for more information on enterprise modeling, and [7] for information on task types). This paper will scrutinize enterprise modeling especially from this perspective of group work and its challenges concerning individual participation, group performance and coordination.

\subsection{Studies on Multi-Touch Use}

There have been several studies dealing with MTTs in general. Especially in educational context, advantages of these devices are underlined. They allow sketching ideas that can be easily changed or erased from screen, thus being less fixed and restricting than notes on paper $[8,9]$. On the other hand, studies report that input via touch keyboard is more laborious and time-consuming [10]. Several studies compared MTTs with other tools assuming an influence of the tool on collaboration. Setting a brainstorming task, [11] discovered that users of MTTs contributed less verbally and in gestures than users of a table covered with paper, but more than users of a flip chart. They hypothesize that the novel medium is distracting and thus restrains collaboration. [12] observed closer collaboration of teams using a MTT compared to pen and paper. When comparing PC and MTT for UML modeling, [13] found the team members' contributions in terms of modeling more evenly balanced and the collaboration to be closer at the MTT. [14] considered laptop and MTT with and without tangible objects where the laptop turned out to cause less evenly distributed verbal contributions. All in all, MTTs seem to be promising for the purpose of participative enterprise modeling. However, several other factors play a role in this, such as the orientation of the medium [15] and the task at hand [11] which may vary in enterprise modeling. 


\section{Method}

\subsection{Experimental Design}

As the influence of the tool on group work was to be explored, an experimental design was applied. The independent variable was represented by the tool which was either whiteboard or MTT. Following a between-subjects design, an enterprise modeling task was to be solved in teams of three either on the whiteboard or the MTT. The team size of three was chosen due to the limited size of the media and because [16] claims that group effects are to be encountered with only a team size of at least three.

The dependent variables corresponded to the participation of the group members, perceived team coordination and organization. The group members' participation was measured based on their contributions in terms of talking and modeling including activities such as writing, drawing and moving elements on the respective medium, e.g. cards on the whiteboard. Participation was assessed via observation using video recordings of the modeling sessions. Perceived coordination and organization among team members were assessed via individual interviews. The participants were asked whether certain team members were responsible for or often took on certain tasks and how such task divisions arose. Moreover they were asked to describe how their group approached the task, with a special focus on modeling activities. Furthermore, group performance was measured by considering the complexity of the final solution in terms of number of components and relations drawn.

\subsection{Procedure}

The study took place at a laboratory of the computer science department at the University of Rostock. The participants were recruited by personal request. The assignment of persons to groups was organized taking the participants' personal schedules into consideration. The groups were assigned to the medium randomly. The enterprise modeling task the participants were expected to perform referred to the modeling of goals and problems of a pizza delivery service, an application field the participants would probably connect with. Moreover, goal and problem models belong to the most basic enterprise models in the 4EM method suggested by [1] which should be easy to apply even for less experienced modelers. It was made sure that at least one team member had at least some experience with the 4EM notation of goal models. If the participants' time schedule allowed it, a face-to-face tutorial was provided before the study in a separate meeting.

At the beginning of each trial, the three participants of a group and the investigator met in the laboratory. Beside the investigator, at least one person in charge of the technical equipment was present, but stayed in the background. In some cases the participants had not met before such that they had to be introduced to each other. Refreshments were provided to create a relaxed atmosphere. Each participant received a handout containing the modeling task and a short reference of the 4EM notation for goal models. They were explicitly asked to work together on the task. In case the group had to work with the MTT, an introduction to the user interface of the self-developed modeling software was given (see right-hand screenshot in Fig. 1). The software provides an editor for $4 \mathrm{EM}$ goal and problem modeling enabling users to model at the same time. Menus can be opened at every spot of the canvas to create components such as goals and problems. All components can be moved and turned at will. If a user wants to add a description to a component, a keyboard will pop up right below the component. Thus, users do not have to share a keyboard; multiple keyboards allow parallel editing. Each keyboard's position depends on the appendant component's position. Components could be linked by drawing arrows. These component relations could be further described by selecting one of the predefined annotations, e.g. "hinders" for a problem hindering a goal. We did not introduce a facilitator because one purpose of the study was to provide insights into natural working behaviors that may help facilitators in chairing modeling sessions.

After the participants had finished reading and remaining questions were answered by the investigator, they started to work on the task. Three groups used a MTT (size: $1210 \mathrm{x}$ 
$680 \mathrm{~mm}$ ) as can be seen in Fig. 1, the other three groups used a whiteboard (size: $2000 \mathrm{x}$ $1000 \mathrm{~mm}$ ). The whiteboard groups were additionally equipped with colored cards, magnets to pin cards to the board, and pens. A time limit of 30 minutes was set for the task. The modeling sessions were video and audio-recorded using two cameras, one installed at the ceiling and another one standing on a tripod. After the task was finished, interviews were conducted with each participant in parallel in separate rooms. The procedure was concluded by letting each participant fill out a questionnaire to capture demographics, experience with modeling notations and use of MTT.

\subsection{Sample}

Overall, 18 persons took part in the study, i.e. three teams using the whiteboard and three teams using the MTT. They were all students of business information systems or computer science. Three participants were female, two of them joined one team that was assigned to the MTT, the third woman joined a team working with a whiteboard. In the whiteboard group, participants were 24.9 years old on average $(\sigma=2.2, \max =28, \min =22)$, in the MTT group, participants were 23.6 years old on average $(\sigma=1.9, \max =28, \min =22)$. The participants came from three different nations. There was one completely Russian team, one completely Indian team and a mixed team of two German students and one Indian student. In two of the teams, the members knew each other already, in one team only two members knew each other before. The whiteboard group comprised two completely German teams whose members all knew each other before, and one mixed team of all three nations whose members had not met before. The level of experience of the 4EM notation, measured with a 5-point scale with 1 representing no experience, was at 2 on average for the MTT group $(\sigma=1, \max =3, \min =1)$ and at 3.7 on average for the whiteboard group $(\sigma=0.6, \max =4, \min =3)$. On a 5-point scale, the participants of the MTT group estimated their experience with MTTs at an average value of 1.7 with 1 representing no experience $(\sigma=1.3, \max =5, \min =1)$.

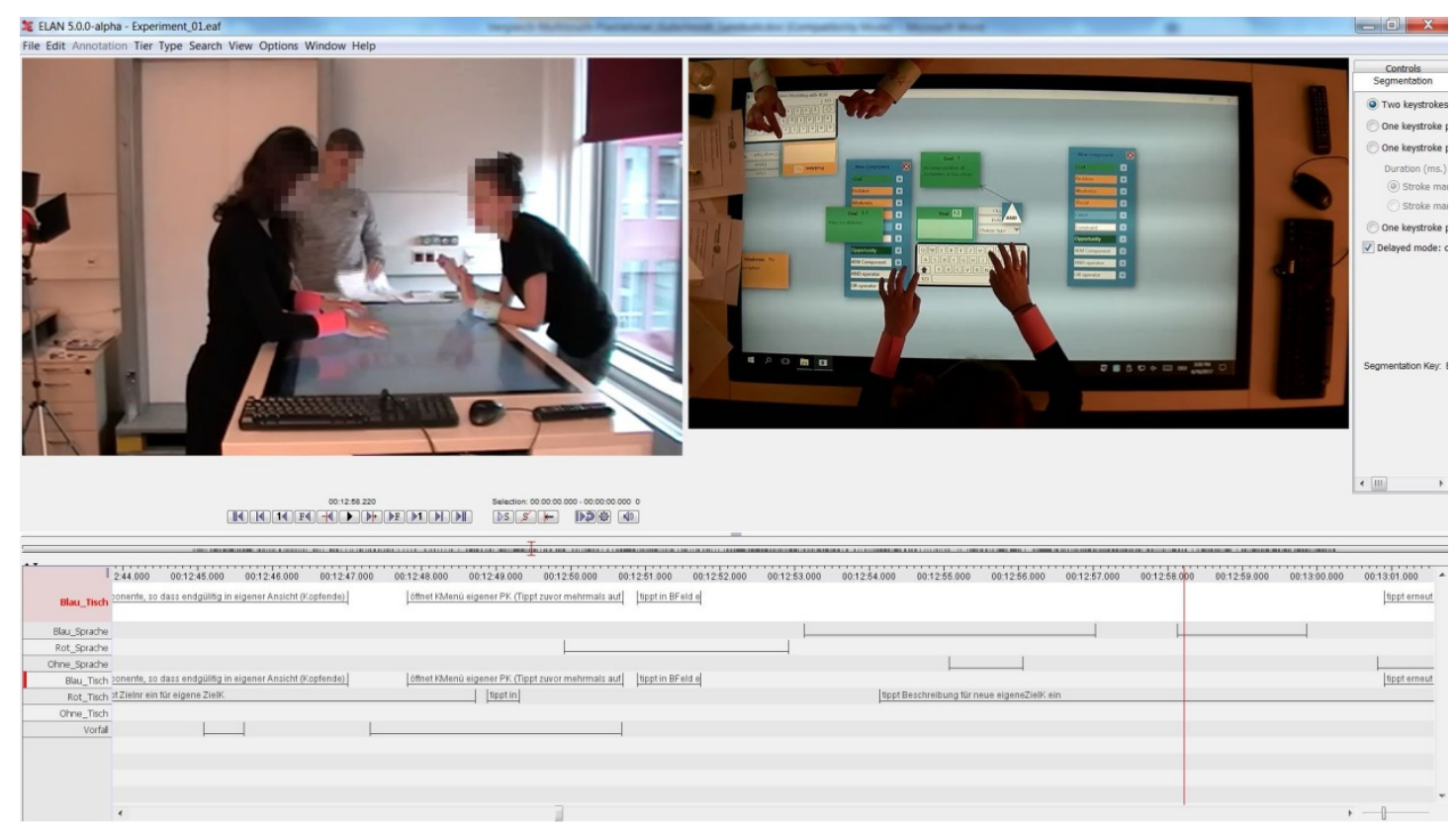

Fig. 1. Marking and annotating activity units in the video recordings with ELAN [17].

\subsubsection{Data Evaluation Methods}

The data from the questionnaires were statistically evaluated using the software SPSS. The interviews were transcribed, and qualitative content analysis according to [18] was applied, supported by the Software MaxQDA. First, the interviews were scanned for statements about 
coordination among team members and the organization of their work. The resulting coding units were inductively ordered into categories.

For the evaluation of the video recording, the software ELAN was used [17]. To determine all time units of talking and modeling of each single person, the respective sections had to be marked on a timeline in the software as Fig. 2 shows. Furthermore, the time units of modeling were annotated with a detailed description of the according activity. In a subsequent step, the activities were again categorized, leading to major activities such as creating a new component (e.g. goal), moving a modeling component over the screen of the MTT or the whiteboard, respectively, writing or drawing relations between components. Each activity was linked with a time stamp such that talking and modeling behavior of each participant in the course of the whole session could be depicted and analyzed. Special focus was put on particular events in the modeling referring to the creation of content (e.g. create component, write, draw relation, pin component). The team members' contributions were determined by considering individual speaking and modeling time in relation to speaking and modeling time of the whole team.

\section{Results}

\subsection{Participation}

Fig. 2 shows the time proportions spent on talking and modeling in separate, and on talking and modeling on the whole for each member of each team. For all members in all groups it was captured when they contributed to the modeling work in a creative way in terms of adding content, comprising the creation of components (e.g. goal), writing, drawing relations between components and pinning components to the whiteboard. For the latter, there is no corresponding event on the MTT. Fig. 3 shows the occurrence of these events in all teams in the course of the modeling session (30 minutes).
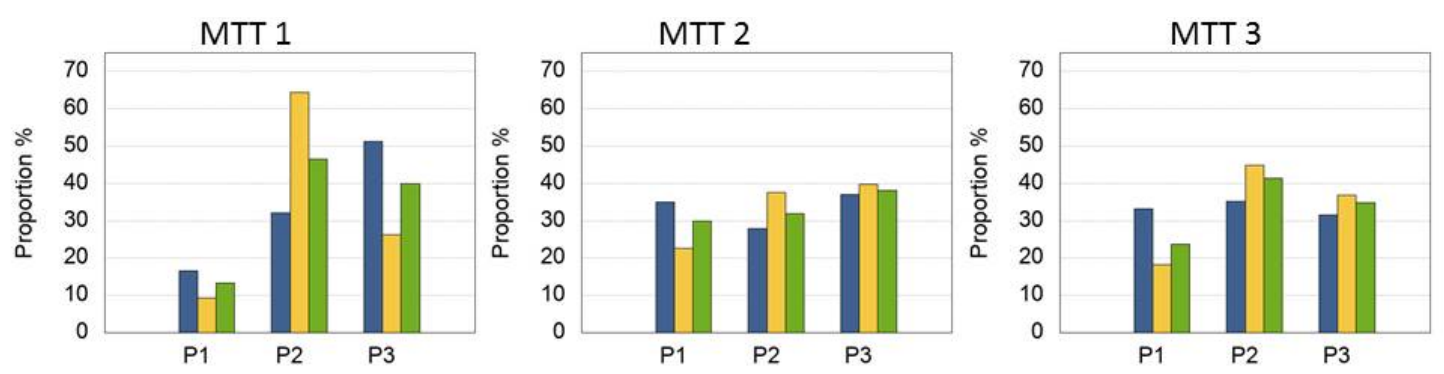

WB 1
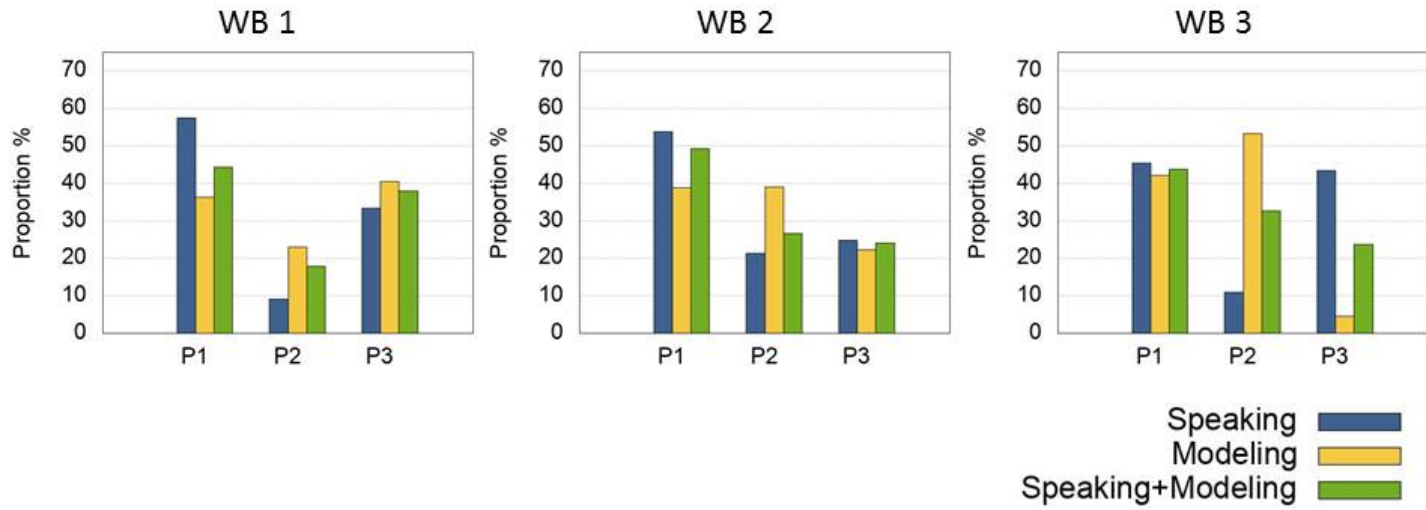

Fig. 2. The time proportions spent by the members $(\mathrm{P})$ of each team on speaking, modeling as well as speaking and modeling in sum; MTT = multi-touch table, WB = whiteboard. 

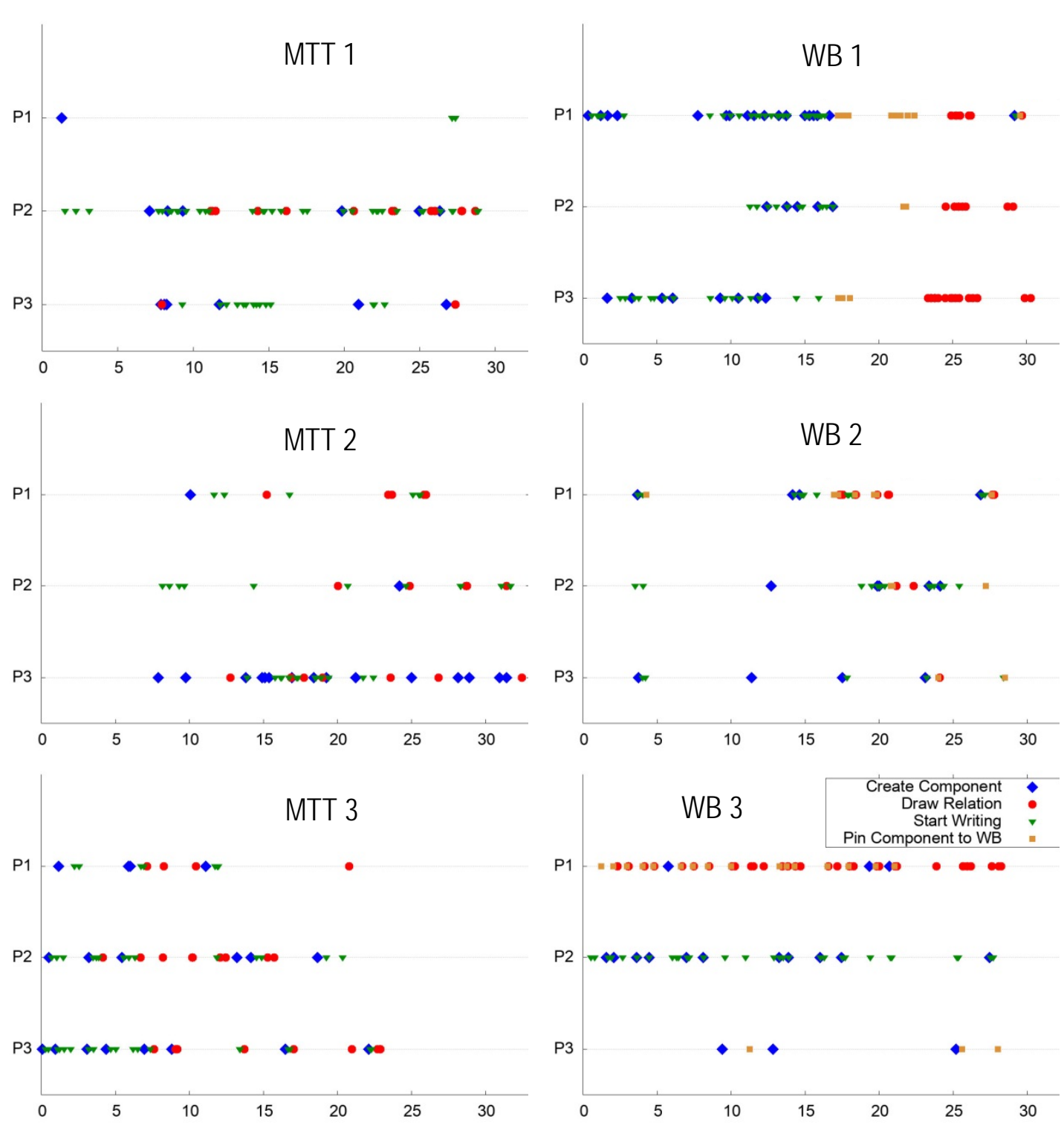

Fig. 3. Modeling events where content was created by the participants in the course of the 30 minute modeling session.

\subsection{Coordination and Organization}

Based on statements on team coordination and organization occurring in the interviews, six major categories arose which deal with (I) the subjects the team members explicitly agreed on, (II) statements that collective approval was part of the proceeding, (III) the existence of task divisions and if so, which form they took, (IV) reasons for certain task distributions, (V) parallel working, and (VI) awareness of the teammates' activities during the task.

\section{Subjects of explicit agreement}

One participant of a team working with a MTT and one participant of a whiteboard group mentioned that the team explicitly agreed on how to start working. E.g. the latter said, "We soon agreed that we would start with goals and then move to problems and then the rest, like adding constraints and opportunities to the model ...” (6,3,1,39, translated from German). ${ }^{1}$ During the

\footnotetext{
${ }^{1}$ Citations from interviews are given with number of trial, number of participant, page and paragraph.
} 
task, some teams consulted about how they would further proceed, as was mentioned by four interviewees from two MTT groups and two whiteboard groups. One participant who had worked with a whiteboard stated that the team had to agree on the level of abstraction with which they approached the task. Three participants from two MTT groups and one from a whiteboard group mentioned that they explicitly agreed on how or when to use the medium. E.g., "They directly wanted to do it on the software, but I convinced them, consoled them like it's better list it first and just go there and put it on" $(3,1,1,11)$.

\section{Getting collective approval}

Two participants of a MTT group mentioned that during their work they constantly searched for a common agreement, e.g. "Before we did anything, we always said first: we can do that" $(4,3,1,37$, translated from German). In each whiteboard group, there was always one person who described a similar behavior.

\section{Division of tasks}

In every group, except for one whiteboard group, at least one team member stated that there was no fixed division of tasks. In most cases, the statements refer to modeling activities, e.g. one participant of a MTT group said: "after that I was writing something, she was writing something, $X X X$ was writing something " $(1,3,1,1)$. In one whiteboard group, one participant said, "No, everyone everything together practically" (6,3,1,41, translated from German) referring to content-related contributions, while his teammate said they had fixed roles with regard to the modeling ("We did not vary. We always had the same roles really", 6,1,1,11, translated from German). Two of those team members described this task division by modeling activity in detail, e.g., "We had this division of tasks, one wrote - meaning on the cards. I pinned them to the thing [whiteboard] and wrote on it. And one commented" (6,1,1,9, translated from German). Some groups, however, used a content-related division of tasks. One whiteboard group divided the task according to component types, as described by all team members, e.g., "... one captures goals, the other one problems, and the other one already opportunities or threats or whatever" (2,1,1,9, translated from German). There were two statements from two MTT groups and two statements from one whiteboard group that team members were responsible for certain topic areas, often corresponding to main goals.

\section{How task distributions arose}

With regard to the reasons why and how certain task distributions arose, some single statements could be found in the interviews. In one MTT group, a participant said that a teammate was mainly drawing the model because this person had more experience with the 4EM notation ("I think that she had the project connected with 4EM and she is more experienced in this case, so we decided that she is right person to do it", 1,3,3,3). One participant who had worked with the whiteboard stated that he "was a bit busy writing" (6,2,1,7, translated from German), so he could not fully contribute to the discussion all the time. Another participant explained that the team members knew each other before such that everyone already had their role in the team $(2,1,1,13)$. In one MTT group, as stated by two of its members, it was seemingly usual that the person who had the idea also wrote it down. Tasks were also distributed by request or enquiry. In one MTT group, all team members describe that teammates had been asked to do something. Two participants from two whiteboard groups described a similar behavior, e.g., "On enquiry and response. Meaning, 'Do you want to write?', 'Yes', and that was it.” (6,3,1,35, translated from German).

In all MTT groups, participants mentioned that the team members' position was a reason why certain persons turned out to be responsible for certain modeling activities. According to one participant, the orientation of the screen played a role ("... we staying the different sides and the first orientation, the right orientation were only for one person, so XXX stayed in the right position and we stayed in the other sides", 1,3,3,3). Two participants said 
that proximity was another reason ("I was doing, because I was [...] right side and the creating menu was the right side. So everytime I was doing this thing.", 3,3,1,16). One of these participants also considered space as important (“... because he always had a lot of space up there in his corner ...”, 4,3,1,65, translated from German). The participant further explained that the keyboards, which were automatically attached to every component as soon as the edit mode was opened, and the menu to create components, of which several instances could be opened everywhere on the screen, restricted the team's space.

Some participants described that the distribution of tasks in their team arose out of its own. One participant from one MTT group and four participants from all three whiteboard groups said that the task distribution emerged without any apparent reason, e.g., "It just happens. “ $(5,2,1,27)$.

\section{Parallel working}

Two participants from two MTT groups mentioned that they worked in parallel. One of them explained further that parallel working concerned modeling activities in particular and that they did not choose to model in parallel at the beginning, but they changed to this mode in the course of the task. This participant and another one from the second aforementioned MTT group also describe that they had been discussing while teammates were modeling at the same time. However, some participants also said that they did not work in parallel. A participant from a whiteboard group even said "when somebody was writing, there was mostly silence during that time " $(6,1,1,23$, translated from German). One MTT group seemed to have had some trouble with the software such that it appeared to them that parallel working was not supported, as mentioned by one member ("Actually, we've tried in the beginning to do at the same time, but the system was not that cooperative for that. We thought of like, we'll do one by one. When one was completed with the goal, and the second person will start at goaling”, 4,1,1,25). The other two team members stated that they changed to non-parallel working particularly later in the process, e.g., "because later at the end when..., there was mostly just one working ..." (4,2,1,17, translated from German).

\section{Awareness}

Two participants of one MTT group mentioned that the MTT helped them to keep an overview of what was going on during the task, e.g., "Once again wrote something, then you looked. What did the other one do? Ah yes, okay, then you can still write that ... " $(4,2,1,11$, translated from German). However, two participants from two whiteboard groups said that there had been a moment where they lost track of what was done by the others, e.g., "where I was concentrated on this and I lost track of what XXX and YYY were doing at the other corner of the whiteboard, what lines they were drawing. And then I confined myself to my right side of the board " (2,3,3,3, translated from German).

\subsection{Group Performance}

Table 1 describes the complexity of the goal models the groups created including the number of components, among them decomposition elements (and, or), the number of relations in general and relations that have been annotated, e.g., a problem may hinder a goal.

Table 1. Parameters describing the complexity of the respective groups' final model.

\begin{tabular}{|l|r|r|r|r|r|r|}
\hline & \multicolumn{1}{|l|}{ MTT 1 } & \multicolumn{1}{l|}{ MTT 2 } & \multicolumn{1}{l|}{ MTT 3 } & \multicolumn{1}{l|}{ WB 1 } & WB 2 & \multicolumn{1}{l|}{ WB 3 } \\
\hline \#Components & 13 & 14 & 17 & 29 & 17 & 19 \\
\hline \#Decompositions & 1 & 1 & 1 & 1 & 3 & 0 \\
\hline \#Relations & 14 & 15 & 20 & 27 & 16 & 31 \\
\hline \#Annotated Rel. & 0 & 13 & 18 & 23 & 0 & 31 \\
\hline
\end{tabular}




\section{Discussion}

\subsection{Summary and Interpretation}

A study was presented that compared the use of MTT and whiteboard in the context of participative enterprise modeling. First, verbal contributions and modeling contributions of the members within each group were analyzed. Fig. 2 gives hint on more evenly distributed participation as the respective charts appear more compact for group MTT 2 and MTT 3. However, looking at modeling events triggered by the individual team members in Fig. 3, the distribution of modeling activities appears less balanced. It seems that with regard to really creating content, one team member was particularly dominating. Addressing our first research question, the differences between the team members' contributions on whiteboard or MTT are not clear enough to lead to definite conclusions. Addressing the second research question on collaboration styles, the most interesting findings concern parallel working, awareness, and reasons for task assignments. Although we did not identify working styles typical of a certain tool, the study shed light on general ways of working that may arise within teams engaged in participative modeling.

In team MTT 1, there is mainly one person performing the modeling activities as this team member is the only one with sound experience in the modeling notation. A second team member, however, is dominating in the discussion whereas the third team member has a low proportion of verbal and modeling contributions.

According to the interviews, teams MTT 2, MTT 3 and WB 2 divided their work among their members based on topics which is reflected in Fig. 3 by the occurrence of all kinds of modeling events for all persons, although not always evenly distributed among team members, and during the whole modeling session without any systematic order. Two members of team MTT 3 mentioned that if a team member presented an idea this person would also model the according content. This led to more even distributions than in teams MTT 2 and WB 2.

The depictions of modeling events are especially characteristic for teams WB 1 and WB 3. In team WB 3, a division of modeling activities arose such that one person wrote on the cards representing model components such as goals and problems, a second person pinned these cards to the whiteboard and drew and annotated relations between the components, and the third person contributed mainly by discussion. On rare occasions, the third team member picked a new card from one of the card stacks only to hand it over to the teammate who had become responsible for writing on the cards, or he pinned a card to the whiteboard, but mostly left this job again to the other teammate who had meanwhile turned out to be responsible for that job. This is underlined by one team member's statement in the interview, describing their roles as fixed.

Team WB 1, the other team that showed a very significant working behavior, decided for a division of work based on component types; i.e. goals, problems, constraints etc. Thus, all kinds of modeling events can be seen for all team members in Fig. 3. The time order in which these events occur is, however, most special. According to the interviews, there must have been an agreement to first gather the model components including their descriptions. Then there must have been a moment when the team decided to pin all the cards to the whiteboard and start drawing and annotating relations between the components. Fig. 3 clearly reflects this time order. A similar procedure was chosen by team MTT 2, but the working behavior looks different in Fig. 3 due to the different tool used. While the members of a whiteboard team could work with and write on the cards independent of the whiteboard, the MTT users must look for other means of sketching ideas. Team MTT 2 decided to take notes on a sheet of paper before they started to model on the MTT. This is reflected by a longer delay of modeling activities for team MTT 2 at the beginning of the session compared to all other teams. The working behavior implies that MTT users should be provided with a possibility of sketching ideas apart from the MTT, whether by means of paper and pen or technologically supported must be discussed and further examined.

Concerning explicit arrangements, there do not seem to be many differences between MTT und whiteboard users, as far as can be concluded from the interviews, e.g. on how to start, or 
how to proceed in the middle of the task. The question of how and when to use the modeling tool seemed to be more explicit for MTT users (mentioned by three users in two groups) than for whiteboard users (mentioned by one user only). Due to the MTT's novelty, users might be more aware of the tool and handling it, and consequently they might be more conscious and careful in the way they use the tool.

The MTT seems to stimulate parallel working in terms of parallel modeling, but even more so in terms of discussing and modeling in parallel. Probably owing to time pressure at the last phase of the session, teams decided for parallel working. Only one team abandoned trying to work in parallel at the MTT. As the software was just a prototypical implementation, its interface might not have responded in the way the users had expected and thus might have made the impression that parallel working was not supported. This shows that parallel working and the challenges connected with it must be especially considered when designing and implementing user interfaces of a MTT modeling editor.

According to the interviews, MTT users might be more easily aware of what their teammates are working on. The horizontal work surface might be a reason for this as claimed by [15]. Two participants from two different whiteboard teams mentioned that there had been a moment during the modeling session when they lost track of what the others were doing. These findings support statements by [19] and [11] that vertical work surfaces are less supportive for collaboration than horizontal. One might also assume that the restricted size of the working surface, which seems at first as a disadvantage of the tool, might also turn out to be an advantage as it is easier to keep an overview of the whole model than on the larger whiteboard.

When asked why certain persons took on a certain task, members of all MTT teams named the position at the MTT as one reason. When a person stood in opposite to the orientation of the user interface, turning the elements meant additional effort. Still, it was observed that such MTT users were nevertheless engaged in modeling. Another participant reported that proximity implied responsibility, e.g. somebody standing near the main menu became responsible for creating new components. This is similar to the findings of [20] and [21] stating that the farther away an MTT user is from a part of the interface the less responsibility he or she feels for it. Moreover, it is possible that teams developed a certain routine in terms of a mental set, also known as cognitive fixedness [22]. Menus could be opened at every point on the surface, so other users would have also been able to open a menu and create new components. Due to a mental set, the team members might have stuck to a strategy they have found to be successful once. However, the interviews reveal that space also played a role in this, and thus space management is another challenge when designing the user interface. Team members took on a certain task because they had more space on the working surface where they stood. As a consequence, they were reluctant to open more menus that would take more space.

When considering team performance (third research question), the final whiteboard models tended to be slightly more complex. However, a reason for this might be that two of the whiteboard teams already knew each other before. One participant said they already knew their roles in the team. Moreover, both these teams had sound experience in the modeling notation they had learned in compulsory courses at the university.

\subsection{Limitations}

When interpreting the findings of this study, certain limitations have to be taken into consideration. The study provides a detailed, mainly qualitative analysis of work behavior in modeling teams. It was meant to explore possible differences in collaboration depending on the modeling tool used. That is why a small sample size of 18 persons assigned to six groups was considered as sufficient. For testing hypotheses and drawing generalizable conclusions, further studies and greater sample sizes are needed. Moreover, samples from different domains would be desirable. The behavior of the participants may of course be influenced by their feeling of being observed which can never be prevented completely. It was expected that the feeling of being observed, particularly via cameras, would be weakened overtime while concentrating on solving the task. To explore different ways of working that arise naturally, possibly induced by 
the different tools, roles, such as domain expert and facilitator, had not been predefined. Future studies may further explore especially the role of facilitator and its tasks. In addition, further kinds of models beside goal models must be examined since they might each require a different extent of creative, knowledge-based, problem-solving activities etc.

All in all, however, the study fulfilled its purpose of giving valuable insight into the procedure of participative enterprise modeling. The next paragraph will give hint on next steps, particularly with regard to research in this area.

\subsection{Implications}

The study has shown that a MTT is a tool well-suited for participative tasks comparable to goal and problem modeling. With MTTs, all stakeholders can potentially participate and even work in parallel. Moreover, awareness of other teammates' activities is higher on the horizontal work surface. For both MTT and whiteboard, we discovered several ways of how teams organize themselves to solve a modeling task. However, which of these work styles is most efficient and convenient for teams? Is it really necessary to strive for most balanced proportions of contributions, e.g. by having every team member talk and model to the same extent? Is it advisable to let everybody model their own ideas to give every team member a chance to equally contribute both to discussion and modeling? Should modeling sessions be organized in phases of collecting ideas and then formalizing these in models? Further research is needed on the influence of working styles on desired outcomes. These would lead to suggestions of how a facilitator should chair a modeling session and when interventions are advisable and when they become counterproductive. Moreover, we intend to improve the modeling software aiming at preventing functional fixedness, better space management and supporting awareness. Although there has been research on the topic of awareness for several years in the area of computer supported work (see e.g. [23], [24]), there is still a need for more knowledge on how to design shared workspaces to promote awareness.

Finally, the desired outcomes are manifold. Complexity and quality of a model represent typical outcome variables. Nevertheless, subjective perceptions such as the team members' satisfaction with the modeling process, their acceptance of and commitment with the models also determine the success of an enterprise modeling project. Thus, we will focus our future research especially on this area.

\section{Acknowledgements}

The author wishes to thank the participants of the study, as well as the members of the chair of Business Information Systems of the University of Rostock for their support and providing the technical equipment. Moreover, the author owes thanks to Sarah Freytag, Pavel Chupryna, Jiawei Yan, and Richard Conradi who helped conducting the study.

\section{References}

1. Sandkuhl, K., et al., Enterprise Modeling: Tackling Business Challenges with the 4EM Method. 2014: Springer Berlin Heidelberg.

2. Stirna, J., A. Persson, and K. Sandkuhl. Participative Enterprise Modeling: Experiences and Recommendations. in Advanced Information Systems Engineering. 2007. Berlin, Heidelberg: Springer Berlin Heidelberg.

3. Frey, D. and H.W. Bierhoff, Sozialpsychologie - Interaktion und Gruppe. 2011: Hogrefe Verlag.

4. von Rosenstiel, L. and F.W. Nerdinger, Grundlagen der Organisationspsychologie: Basiswissen und Anwendungshinweise. 2011: Schäffer-Poeschel.

5. Karau, S.J. and K.D. Williams, Social loafing: A meta-analytic review and theoretical integration. Journal of Personality and Social Psychology, 1993. 65(4): p. 681-706. 
6. Lamm, H. and G. Trommsdorff, Group versus individual performance on tasks requiring ideational proficiency (brainstorming): A review. European Journal of Social Psychology, 2006. 3(4): p. 361-388.

7. Steiner Ivan, D., Models for inferring relationships between group size and potential group productivity. Behavioral Science, 2006. 11(4): p. 273-283.

8. Piper, A.M. and J.D. Hollan, Tabletop displays for small group study: affordances of paper and digital materials, in Proceedings of the SIGCHI Conference on Human Factors in Computing Systems2009, ACM: Boston, MA, USA. p. 1227-1236.

9. Mercier, E. and S. Higgins, Creating joint representations of collaborative problem solving with multi-touch technology. Journal of Computer Assisted Learning, 2014. 30(6): p. 497-510.

10. Basheri, M., Multi-Touch Table for Enhancing Collaboration during Software Design in Durham University, 2013.

11. Buisine, S., et al., How do interactive tabletop systems influence collaboration? Computers in Human Behavior, 2012. 28(1): p. 49-59.

12. Basheri, M. and L. Burd. Exploring the significance of multi-touch tables in enhancing collaborative software design using UML. in 2012 Frontiers in Education Conference Proceedings. 2012.

13. Basheri, M., L. Burd, and N. Baghaei, A multi-touch interface for enhancing collaborative UML diagramming, in Proceedings of the 24th Australian ComputerHuman Interaction Conference2012, ACM: Melbourne, Australia. p. 30-33.

14. Rogers, Y., et al., Equal Opportunities: Do Shareable Interfaces Promote More Group Participation Than Single User Displays? Human-Computer Interaction, 2009. 24(12): p. 79-116.

15. Rogers, Y. and S. Lindley, Collaborating around vertical and horizontal large interactive displays: which way is best? Interacting with Computers, 2004. 16(6): p. 1133-1152.

16. Nerdinger, F.W., D. von der Oelsnitz, and J. Weibler, Grundlagen des Verhaltens in Organisationen. 2012: Kohlhammer.

17. Wittenburg, P., et al. ELAN : a professional framework for multimodality research. in 5th International Conference on Language Resources and Evaluation (LREC 2006). 2006. Genoa.

18. Mayring, P., Qualitative Inhaltsanalyse. Grundlagen und Techniken. Beltz Deutscher Studien Verlag, 2008. 6.

19. Muller-Tomfelde, C., A. Wessels, and C. Schremmer. Tilted tabletops: In between horizontal and vertical workspaces. in 2008 3rd IEEE International Workshop on Horizontal Interactive Human Computer Systems. 2008.

20. Scott, S.D. and S. Carpendale, Theory of Tabletop Territoriality, in Tabletops Horizontal Interactive Displays, C. Müller-Tomfelde, Editor. 2010, Springer London: London. p. 357-385.

21. Ryall, K., et al., Exploring the effects of group size and table size on interactions with tabletop shared-display groupware, in Proceedings of the 2004 ACM conference on Computer supported cooperative work, 2004, ACM: Chicago, Illinois, USA. p. 284293.

22. Myers, D.G., et al., Psychologie. 2015: Springer Berlin Heidelberg.

23. Dourish, P. and V. Bellotti, Awareness and coordination in shared workspaces, in Proceedings of the 1992 ACM conference on Computer-supported cooperative work, 1992, ACM: Toronto, Ontario, Canada. p. 107-114.

24. Isenberg, P. and D. Fisher, Collaborative Brushing and Linking for Co-located Visual Analytics of Document Collections. Computer Graphics Forum, 2009. 28(3): p. 10311038. 